\title{
Electron beam polarization with the Compton Polarimeter at JLab
}

\author{
T. Pussieux* for the Compton Polarimeter collaboration \\ *CEA Saclay, DAPNIA/SPhN, 91191 Gif sur Yvette Cedex, France. \\ http://www.jlab.org/compton
}

\begin{abstract}
We built and operated a new kind of Compton polarimeter to measure the electron beam polarization of the Thomas Jefferson National Accelerator Facility (Virginia, USA) to $3 \%$ total error within an hour. The heart of this polarimeter is the coupling of a High Finesse monolithic Fabry-Pérot cavity to the particle accelerator. Its purpose is to amplify a primary $300 \mathrm{~mW}$ laser beam to increase the luminosity at the Compton interaction point. The measured Finesse and amplification gain of the cavity are $F=26000$ and $G=7300$. We have used this facility during the HAPPEX (April-July 1999) experiment.
\end{abstract}

\section{INTRODUCTION}

Among all the possibilities to measure the polarization of an electron beam, (Mott and Moller polarimeters), Compton polarimetry presents the advantage of providing a measurement while the main experiment is running. Our group has completed the installation of the Jlab Hall A Compton polarimeter in feb 1999. It has given its first results for the HAPPEX experiment [1] with a $4 \mathrm{GeV}$ electron beam of $50 \mu \mathrm{A}$. Whereas Compton polarimeters operating at higher energies (SLAC, HERA) or higher currents (NIKHEF) use a high power laser photon source, the challenge of the Compton polarimeter at Jlab was to operate a Fabry-Perot cavity to amplify the photon beam in order to achieve a statistical precision of $1 \%$ within 1 hour.

\section{THE COMPTON POLARIMETER}

The hearth of this polarimeter is a high power $\left(P_{L} \simeq 1.4 k W\right)$ Fabry-Perot cavity injected by a $300 \mathrm{~mW}$ NdYAG infra-red laser [2]. The light inside the Cavity is circuraly polarized, and its helicity can be reversed thanks to a rotatable $\lambda / 4$ plate. The polarization for both helicity is $P_{\gamma}^{R, L}= \pm 99.3 \pm 1.1 \%$ [3].

A magnetic chicane steers the electron beam to the center of the optical Cavity. Backscattered photon are dectected by a $\mathrm{PbWO}_{4}$ calorimeter [4]. Counting rates for events with photon energy $k^{\prime}$ above $k_{s}{ }^{\prime} \simeq 30 \mathrm{MeV}$ are recorded by a scaler. For 
a small fraction of events $(1 \%)$, the energy deposited in the calorimeter $k_{r}^{\prime}$ is also measured thanks to a charge ADC.

The beam polarization $P_{e}$ is extracted from the Compton experimental asymmetry, $A_{e x p}$, via $P_{e}=\frac{1}{P_{\gamma}<A_{c}>} A_{e x p}$, where $\left\langle A_{c}>\right.$ is the analyzing power (A.P.). In practise we measure a raw conting rate asymmetry $A_{\text {raw }}=\frac{r^{+}-r^{-}}{r^{+}+r^{-}}$given by

$$
A_{\text {exp }}=\left[\left(1+\frac{B}{S}\right) A_{\text {raw }}+\frac{B}{S} A_{B}+A_{F}^{p}\right] .
$$

In order to measure the background over signal ratio $\frac{B}{S} \simeq 0.3$ and the background asymmetry $A_{B}$, we take data alternatively with the cavity on ( $\simeq 5$ minutes) and off ( $\simeq 2$ minutes). To reduce systematic errors associated with the luminosity asymmetries $A_{F}^{p}$, the photon polarization is set alternativly right or left.

In addition to the determination of the Light polarisation, the two steps to extract the polarization are the determinaitions of the experimental asymmetry $A_{\text {exp }}$ from the measured raw asymmetry $A_{\text {raw }}$ and of the analyzing power $\left\langle A_{c}\right\rangle$.

\section{MEASUREMENT OF THE EXPERIMENTAL ASYMMETRY $A_{E X P}$}

For each photon helicity state $(R, L)$, a raw asymmetry $A_{\text {raw }}^{R, L}$ is determined using the measured rates (normalized to the beam intensity) $\left(r_{1}^{R, L}\right)$ at $30 \mathrm{~Hz}$ when the cavity is $\mathrm{On}$. If all the parameters were stable between two consecutive photon helicity, then averaging the two raw asymmetries $A_{\text {raw }}^{R, L}$ cancels out the false asymmetries (background and luminosity)

$$
A_{\text {exp }} \simeq\left(1+\frac{B}{S}\right) \frac{1}{2}\left[A_{\text {raw }}^{R}-A_{\text {raw }}^{L}\right] .
$$

The background dilution was estimated by using the rate $r_{0}$ measured when the cavity is off $1+\frac{B}{S}=\frac{r_{1}}{r_{1}-r_{0}}$. With this method, we have measured experimental asymetries close to $A_{e x p} \simeq 1.2 \%$ with a relative statistical error of $1.4 \%$ for a typical one hour run. Variations with time of the rates and the background asymmetry result in a contribution to the relative systematic error of $0.5 \%$ for the dilution and $0.5 \%$ for the background asymmetry.

There is also a contribution to the systematic error from the non complete cancellation of the luminosity asymmetry between the two photon helcity states. False luminosity asymmetry can be traced back to the fact that we are crossing two beams with small transverse sizes $(\simeq 100 \mu \mathrm{m})$ in the vertical direction $(y)$. We thus expect our luminosity to be very sensitive to the vertical electron beam position. For a non optimal crossing (e.g. off-centering by $50 \mu \mathrm{m}$ ), the luminosity exhibits a sensitivy to the electron beam vertical position $\frac{1}{r} \frac{\partial r}{\partial Y}$ that can reach the $0.1 \% / \mu$ 
level. This effect, combined with the fact that there might be systematic different beam positions for the two electron helcity $\Delta y=y^{+}-y^{-}$of the order of $100 \mathrm{~nm}$, could results in a relative false asymmetry close to $1 \%$. To keep this systematics at a small level, roughly $3 \%$ of the data for which the beams were off-centered by more than $50 \mu$ were rejected. Again, in practise, a false asymmetry is computed for each photon polarization state

$$
A_{p}^{R, L}=\sum_{p=x, y, \theta_{a}, \theta_{y}}\left(\frac{1}{r} \frac{\partial r}{\partial p}\right)^{R, L} \Delta p^{R, L}
$$

and the residual systematic error to the experimental asymmetry using Eq. 2 is given by $A_{p}^{F} \simeq 1 / 2\left(A_{p}^{R}-A_{p}^{L}\right)$. This is the largest contribution $(\simeq 1.2 \%)$ to the relative systematics error on the experimental asymmetry.

\section{DETERMINATION OF THE ANALYZING POWER}

In terms of backscattered photon energy $k^{\prime}$, the A.P. is defined by

$$
<A_{C}>=\frac{\int_{0}^{k_{\text {max }}^{\prime}} \epsilon\left(k^{\prime}\right) \frac{d \sigma_{0}^{+}}{d k^{\prime}}-\int_{0}^{k_{\text {max }}^{\prime}} \epsilon\left(k^{\prime}\right) \frac{d \sigma^{-}}{d k^{\prime}}}{\int_{0}^{k_{\text {max }}^{\prime}} \epsilon\left(k^{\prime}\right) \frac{d \sigma_{c}^{+}}{d k^{\prime}}+\int_{0}^{k_{\text {max }}^{\prime}} \epsilon\left(k^{\prime}\right) \frac{d \sigma_{\sigma}^{-}}{d k^{\prime}}}
$$

where $k_{\max }^{\prime}=190 \mathrm{MeV}$ is the Compton edge, $\epsilon\left(k^{\prime}\right)$ is the calorimeter efficiency and $\frac{d \sigma_{c}^{ \pm}}{d k^{\prime}}$ the known Compton helcity dependent cross-sections [2]. In practise, we only measure the energy deposited in the calorimeter $k_{r}^{\prime}$, which differs from the backscattered photon energy $k^{\prime}$ for various reasons (statistical fluctuations, nonlinearity, ...). The A.P. has thus to be computed using this measured energy

$$
<A_{C}>=\frac{\int_{0}^{\infty} \epsilon\left(k_{r}^{\prime}\right) \frac{d \sigma_{c}^{+}}{d k_{r}^{\prime}}-\int_{0}^{\infty} \epsilon\left(k_{r}^{\prime}\right) \frac{d \sigma_{c}^{-}}{d k_{r}^{\prime}}}{\int_{0}^{\infty} \epsilon\left(k_{r}^{\prime}\right) \frac{d \sigma_{c}^{+}}{d k_{r}^{\prime}}+\int_{0}^{\infty} \epsilon\left(k_{r}^{\prime}\right) \frac{d \sigma_{c}^{-}}{d k_{r}^{\prime}}}
$$

In order to determine the dependance of the cross-section $\frac{d \sigma_{c}^{ \pm}}{d k_{r}^{\prime}}$ and the efficiency $\epsilon$ with the reconstructed energy $k_{r}^{\prime}$ we use the background substracted ADC spectrum. This spectrum is first corrected for the non-linearity of the electronics, and then calibrated in energy using the compton edge to get the energy reconstructed spectrum. We then fit this energy reconstructed spectrum by $p_{\left(k_{r}^{\prime}, \sigma_{r}^{s}\right)}\left(k_{r}^{\prime}\right)\left[\frac{d \sigma_{c}}{d k^{\prime}} \otimes g_{(a, b, c)}\left(k^{\prime}\right)\right]$ where the Compton cross-section $\frac{d \sigma_{c}}{d k^{\prime}}$ is convoluted by a gaussian resolution $g_{(a, b, c)}$ with $\sigma=a \oplus b / \sqrt{k^{\prime}} \oplus c / k^{\prime}$ and the calorimeter efficiency is parametrized by an erf function centered around the threshold $k_{r}^{\prime s}$ with a slope proportionnal to $\sigma_{r}^{s}$. With this procedure we extract for each run the A.P., $<A_{C}>\simeq 1.7 \%$. To estimate the systematic error associated with this procedure, we allow the parameters of the fit (e.g the three parameters for the resolution 


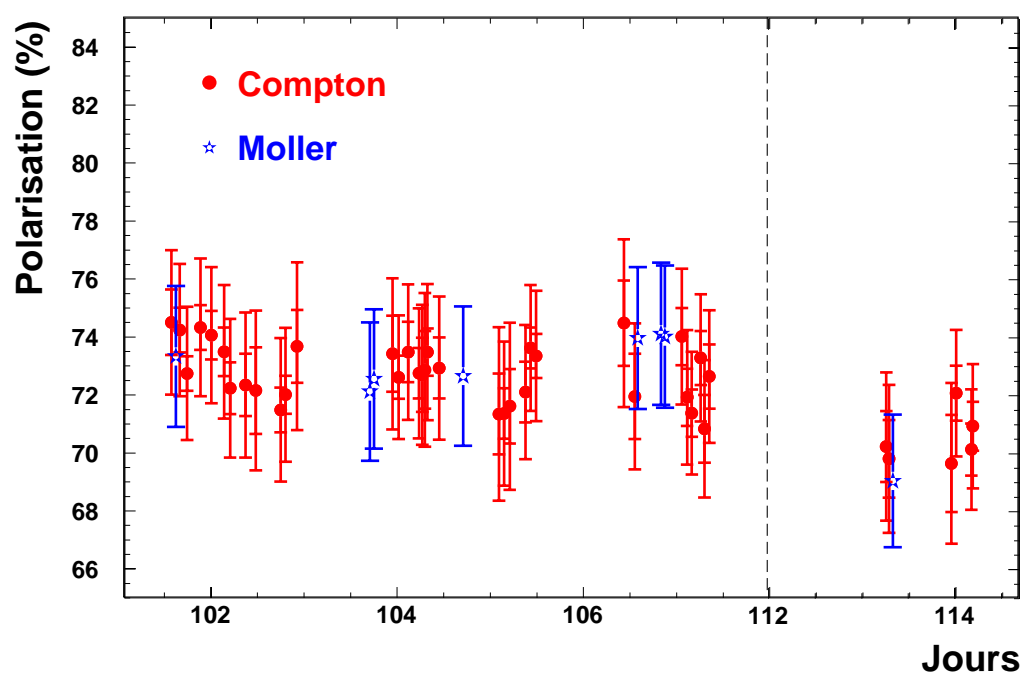

FIGURE 1. Compton and Møller measurement as a function of time.

$(a, b, c)$ and the two parameters of the response function $\left.k_{r}^{\prime s}, \sigma_{r}^{s}\right)$ to vary within a large range and assign as a systematic error on the A.P. the maximum absolute difference between the A.P. determined using the nominal parameters of the fit and the ones determined with each combinaition of the parameters in the above range. This contributes to a relative systematics errors of $1.9 \%$, and is largely dominated by the sensitivity of the A.P. to the threshold value. Eventually, we have added a $1 \%$ contribution to the relative systematic error associated with the non-linearity of the electronics, along with a $1 \%$ contribution to take into account the calibration procedure. This ends up with a total relative systematics errors of $2.4 \%$ associated to the A.P.

\section{RESULTS}

The 40 polarization measurement are given on figure 1 . There are in good agreement with the Møller measurements. The typical total error is around $3.3 \%$. In addition to these absolute measurements, the Compton was able to monitor the polarization at the $1.5 \%$ and ruled out possible large variation of the polarization between two Moller measurements.

\section{REFERENCES}

1. HAPPEX Collab (K.A. Aniol et al.) Phys.Rev.Lett. 82 :1096 -1100 (1999)

2. J. P. Jorda et al., Nucl. Instrum. Meth. A412 (1998) 1.

3. N. Falletto, PhD Thesis, Grenoble University, (1999), unpublished.

4. D. Neyret et al., ton polarimeter," Nucl. Instrum. Meth. A443, 231 (2000) 